\title{
Initial production and fruit quality of scion-rootstock combinations in orange trees in Amazonas
}

\author{
Jefferson Costa Santos ${ }^{1}$, Cláudio Luiz Leone Azevedo ${ }^{2}$, José Eduardo Borges de Carvalho \\ Silvana Pimentel de Oliveira ${ }^{4}$, Ajax de Souza Ferreira ${ }^{5}$, José Ferreira da Silva ${ }^{6}$

\begin{abstract}
The cultivation of orange trees in Amazonas, Brazil relies predominantly on grafted plants combining 'Pera' scion and 'Rangpur' lime rootstock. Alternative rootstocks better adapted to the conditions of the region have not been investigated. Therefore, the objective of this research was to test seven rootstocks for the production of high-quality oranges under the typical conditions of the Amazon. Citrus production was evaluated from May to August between 2017 and 2019, based on the variables of cumulative yield, yield efficiency, and crop-yield index over the study period. The fruit physicochemical quality was evaluated by quantification of total soluble solids, total titratable acidity, ratio, juice percentage, and technological index. 'Indio, 'BRS Bravo', and 'Riverside' genotypes combined with 'Pera'/CNPMF scion had the highest yield and good fruit quality. They are potentially the most suitable combinations to grow sweet oranges in the Amazon. Index Terms: orange trees, Amazon, rootstocks, initial production, quality.
\end{abstract}

\section{Produção inicial e qualidade de frutos de combinações de copas porta-enxertos de laranjeiras no Amazonas}

Corresponding author: jfsilva@ufam.edu.br

Received: December 04, 2020 Accepted: March 23,21

Copyright: All the contents of this journal, except where otherwise noted, is licensed under a Creative Commons Attribution License.

\begin{abstract}
Resumo - O cultivo de laranjeiras com predominância da combinação 'Pera' sobre limoeiro 'Cravo' nos plantios de citros no Amazonas, que possui potencial de produção para a fruteira, deve-se à ausência de estudos que indiquem porta-enxertos alternativos adaptados às condições da região. O objetivo do trabalho foi testar sete porta-enxertos para a produção e a qualidade de laranjas em condições Amazônicas. A avaliação da produção foi realizada no período de maio a agosto, nos anos de 2017 a 2019. A avaliação da produção foi realizada levando-se em consideração as variáveis produção e produtividade acumuladas, a eficiência produtiva e o índice de produtividade ao longo do período de estudo. A avaliação da qualidade físico-química dos frutos foi feita pela quantificação de Sólidos Solúveis (SS), Acidez Total (AT), Ratio, porcentagem de suco e índice tecnológico. Os genótipos 'Indio', BRS Bravo e 'Riverside', em combinação com a variedade copa 'Pera'/CNPMF, apresentaram maior produção, além de boa qualidade de frutos. Por tratar-se de um trabalho-piloto com produção inicial, estas serão, potencialmente, as combinações mais indicadas.
\end{abstract}

Termos para indexação: laranjeiras; Amazônia; porta-enxertos; produção inicial; qualidade.

${ }^{1} \mathrm{PhD}$ Student in Tropical Agronomy at Federal University of Amazonas. Manaus. Amazonas. Brazil. E-mail: jefferson.doutorando@gmail.com ${ }^{\text {(ORCID }}$ 0000-0001-9288-5034)

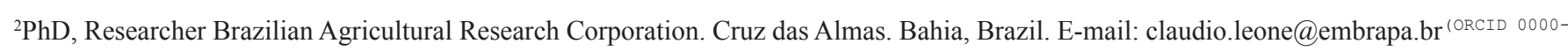
0001-7698-5051)

${ }^{3} \mathrm{PhD}$, Researcher Brazilian Agricultural Research Corporation. Cruz das Almas. Bahia, Brazil. E-mail: jose-eduardo.carvalho@embrapa.br ${ }^{(0 R C I D}$ 0000-0002-5715-2924)

${ }^{4} \mathrm{PhD}$ Student in Tropical Agronomy at Federal University of Amazonas. Manaus. Amazonas .Brazil. E-mail: silvanapimentel.oli@gmail.com ${ }^{\text {(ORCID }}$ 0000-0003-1218-3134)

${ }^{5}$ Master student in Tropical Agronomy at Federal University of Amazonas. Manaus. Amazonas. Brazil. E-mail: ajaxsousa@yahoo.com.br ${ }^{\text {(ORCID }}$ 0000-0003-3568-0790)

${ }^{6} \mathrm{PhD}$, Full Professor at Federal University of Amazonas. Manaus. Amazonas. Brazil. Faculty of Agrarian Sciences. E-mail: jfsilva@ufam.edu.br ${ }^{(0 R C I D}$ 0000-0001-6545-9216) 


\section{Introduction}

In Brazil, citrus production is distributed across all regions, with a higher prevalence in the southeast. In northern Brazil, citrus farming has grown in recent years, especially in the Amazonas and Pará states, which had the largest share of orange production in the region between 2017 and 2019 (IBGE, 2019). Citrus production in Amazonas increased from 55,837 to 71,830 tons between 2015 and 2017. In 2019, citrus production in Amazonas was approximately 66,756 tons (IBGE, 2019); 5,074 tons fewer than in 2017, but 10,919 tons more than in 2015 . From 2015 to 2019, Amazonas produced an average of $20,032.60 \mathrm{~kg} \cdot$ ha $^{-1}$ oranges per year, reaching productivity levels similar to Minas Gerais, the state with the secondhighest orange production, averaging 23,510.08 kg.ha-1 (SIQUEIRA; SALOMÃO, 2017). Bahia and Sergipe averaged $14,859.67$ and $11,504.83 \mathrm{~kg} \mathrm{ha}^{-1}$, respectively, during that same period (IBGE, 2019). Amazonas is a promising citrus producer, however, adapted scions and rootstocks are needed to increase fruit productivity and quality in the region.

The fastest and most effective strategy to obtain cultivars with superior fruit quality is introducing germplasm to a location, evaluating its performance, and subsequently selecting plants with desirable and adapted characteristics (NEGREIROS et al., 2014). Most orchards employ grafted trees, combining the favorable attributes of scion and rootstocks (CARVALHO et al., 2016). The number of orange scion varieties grown in Brazilian orchards is small, and even fewer rootstocks are routinely used; grafted trees mostly apply 'Rangpur' lime Citrus limonia (L.) Osbeck as rootstock (FRANÇA et al., 2018; RODRIGUES et al., 2019; RIBEIRO et al., 2020; RODRIGUES et al., 2020). This reduced diversity, which also occurs in Amazonas, is a vulnerability of the citrus production chain because it increases susceptivity to biotic and abiotic threats. Here, we aimed to identify the best alternative rootstocks to 'Rangpur' lime for citrus cultivation in the Amazon region by evaluating the effect of seven rootstocks on the production and physicochemical quality of four orange varieties.

\section{Material and Methods}

The experiment was installed at the beginning of 2013 and evaluated from 2016 to February 2019 in the municipalities of Rio Preto da Eva and Manaus, AM, Brazil, at the geographic coordinates $02^{\circ} 37^{\prime} 05.02^{\prime \prime} \mathrm{S}$ $59^{\circ} 40^{\prime} 53.87^{\prime \prime} \mathrm{W}$ and $02^{\circ} 51^{\prime} 17.24^{\prime \prime} \mathrm{S} 60^{\circ} 02^{\prime} 24.28^{\prime \prime} \mathrm{W}$. The soil of the study areas was classified in the Latosol and Argisol orders. The experiment was performed in two areas, in a total of 3.43 ha. Soil tillage for crop implementation included harrowing, subsoiling, and in-depth application of gypsum. The nursery trees were planted in $40 \times 40 \times 40 \mathrm{~cm}$ pits, opened with a drill, spaced at $6.0 \times 2.5 \mathrm{~m}$, and with a density of 667 trees.ha $^{-1}$.

Seven rootstocks were used with four scions in a total of 28 combinations. The rootstocks tested included 'Rangpur' lime, 'Sunki Tropical' mandarin [Citrus sunki (Hayata) hort. ex Tanaka], 'Indio' and 'Riverside' citrandarins (Poncirus trifoliata hybrids), both from the Indio experimental station in California, and the hybrids BRS Pompeu, LVK x LCR - 038, and 'BRS Bravo' from the Brazilian Agricultural Research Corporation Cassava and Fruit. The Citrus sinensis (L.) Osbeck scions tested were 'Rubi', 'Pera'/CNPMF,' 'Valencia Tuxpan,' and 'Pineapple.'

\section{Production}

Production was evaluated from 2017 to February 2019 based on yield weight, cumulative yield (CY), yield efficiency (YE), and crop-yield index (CYI). The fruits were weighed on a $20 \mathrm{~kg}$ pan balance to determine the yield in $\mathrm{kg}$ per plot. The experiment was set up with five trees per plot. Afterward, the yield per plant was calculated by dividing the total yield $(\mathrm{kg})$ in each plot by the number of trees in the plot ( $\mathrm{kg} /$ tree $)$. The CY was calculated by converting yield per tree $(\mathrm{kg} /$ tree $)$ into yield in t.ha ${ }^{-1}$ using the conversion factor of 0.677 . Yield and CY were calculated by adding yield values per tree in each harvest of each year.

The YE, which compares plant fruit production efficiency to its vegetative development, was calculated as the ratio between accumulated production $(\mathrm{kg})$ and scion volume $(\mathrm{CV})$, according to the formula $\mathrm{YE}\left(\mathrm{m}^{3}\right)=2 / 3$ $\pi R^{2} H$, where $\mathrm{R}$ is the crown radius, $\mathrm{H}$ is the plant height, and $\pi$ equals 3.14 . The CYI represents the relationship between the $\mathrm{CY}$ and the area of the trunk section (BLUMER, 2005); it is calculated by the formula CYI= $\mathrm{CY} /$ trunk section area. The trunk section area is calculated by $\mathrm{A}=\pi \mathrm{R}^{2}$. Measurements were made $5 \mathrm{~cm}$ above and $5 \mathrm{~cm}$ below the grafting point, using a measuring tape to determine the mean perimeter and mean radius of the trunk section. The mean trunk radius estimate was calculated using the equation: $R=P m / 2 \pi$, where $R$ is the mean radius of the trunk section, $\mathrm{Pm}$ is mean trunk perimeter, measured $5 \mathrm{~cm}$ above and $5 \mathrm{~cm}$ below the rootstock, and $\pi$ equals 3.14 . 


\section{Fruit quality}

Samples of fifteen fruits and their respective juice were weighted to determine fruit juice content (JC) and the yield of each plot. Juice and fruit weights were measured on a $20 \mathrm{~kg}$ pan balance, following the methodology applied by Petry et al. (2015), and according to the formula $\mathrm{JC}(\%)=($ juice weight $/$ weight of fifteen fruits $) \times 100$. The production of juice concentrate was determined by quantifying the raw material with the technological index (TI), calculated with the following expression: $\mathrm{TI}=(\mathrm{B} \times$ J) / 100, where B represents the total soluble solids (TSS) and $\mathrm{J}$ is the JC (CHITARRA;CHITARRA, 2005).

Chemical analyses were performed by directly measuring TSS content in ${ }^{\circ}$ Brix using a refractometer. Total titratable acidity (TTA) was determined by titrating the orange juice sample with $0.1 \mathrm{~N} \mathrm{NaOH}$, and expressed in citric acid percentage. The ratio was determined by dividing TSS by TTA.

\section{Statistical Analysis}

The collected data underwent analyses of variance using the Assistat 7.0 software; the data were transformed into $\sqrt{\mathrm{x}}$ for all data that did not follow a normal distribution.

All the variables underwent multivariate analysis to identify relatively homogeneous groups of scion-rootstock combinations. For this purpose, the multivariate Principal Component Analysis (PCA) method was applied to assess variability and relationships between the variables. The scion-rootstock combinations were clustered with the Hierarchical Cluster Analysis method using the Euclidean distance as a measure of dissimilarity and Ward's method for connection. These analyses were performed using the Origin 2019b software.

\section{Results and Discussion}

\section{Yield, cumulative yield, yield efficiency, and crop-yield index}

We found statistically significant differences between the $\mathrm{CY}$ values of the various rootstock-scion combinations analyzed from 2016 to 2019 (Table 1); fruit production only occurred from 2017 to 2019 . The highest cumulative yields occurred with 'Indio' citrandarin, 'BRS Bravo', and 'Riverside' citrandarin rootstocks combined with 'Pera'/CNPMF. The scion variety 'Pineapple' had a lower yield when grafted on these rootstocks; the same behavior was found in 'Valencia Tuxpan' $x$ 'Indio' citrandarin (Table 1).
The highest yield rates were obtained with 'Pera'/ CNPMF combined with 'Indio' citrandarin followed by 'Pera'/CNPMF combined with 'Riverside' citrandarin. The 'Pineapple' scion combined with 'Indio,' 'Riverside,' and BRS Bravo was the second most efficient variety even when using 'Riverside' and 'BRS Bravo' with this variety (Table 1). The 'Valencia Tuxpan' scion had similar YE and yield rates when grafted onto 'Sunki Tropical,' 'Indio,' and 'Riverside,' but the CY values differed with the rootstock. The highest CY of the 'Valencia Tuxpan' scion was achieved when grafted onto 'Indio' (Tables 1 and 2). Finally, similar results for YE were obtained with the 'Valencia Tuxpan' scion grafted onto 'Indio,' 'Riverside,' and BRS Bravo (Table 1).

\section{Fruit quality}

There were significant differences in all chemical quality variables determined for the four scion and seven rootstock combinations (Table 3). The 'Rubi' fruits had high TSS content, ratio, and TTA when combined with most rootstocks. Furthermore, they had the second highest TI in combination with 'BRS Bravo' and 'Riverside.' The 'Pera'/CNPMF x 'Sunki Tropical' combination resulted in the highest fruit TSS content (Table 3). In general, the 'Valencia Tuxpan' led to high JC and low TTA, but lower TSS levels than 'Pineapple,' 'Rubi,' and 'Pera'/CNPMF. The 'BRS Bravo' rootstock combined with 'Pineapple' led to low TTA, high TSS content, and a high TI. The 'Rangpur' lime, 'Sunki Tropical,' and 'Indio' rootstocks also led to low TTA and high Ratio values in the tested scions (table 3 ). 
Table 1. Cumulative yield (CY), crop-yield index (CYI), and yield efficiency (YE) of the combinations of seven rootstocks with four crowns tested in the Manaus metropolitan region, AM, from 2017 to 2019.

\begin{tabular}{|c|c|c|c|}
\hline Combinations & CY $\left(\right.$ t.ha $\left.{ }^{-1}\right)$ & CYI $\left(\mathrm{kg} \cdot \mathrm{cm}^{-2}\right)$ & $\mathrm{YE}\left(\mathrm{kg} \cdot \mathrm{m}^{-3}\right)$ \\
\hline 'Rubi'x 'Santa Cruz Rangpur' lime & $0,195 \mathrm{j}$ & $0,80 \mathrm{e}$ & $0,93 \mathrm{e}$ \\
\hline 'Rubi’x 'Sunki Tropical' & 0,1601 & $0,77 \mathrm{e}$ & $0,83 \mathrm{e}$ \\
\hline 'Rubi’x x 'Indio' & $1,212 \mathrm{f}$ & $1,28 \mathrm{c}$ & $1,33 \mathrm{c}$ \\
\hline 'Rubi’ x 'Riverside' & $0,970 \mathrm{~g}$ & $1,29 \mathrm{c}$ & $1,36 \mathrm{c}$ \\
\hline 'Rubi' x BRS Pompeu & 0,1091 & $1,15 \mathrm{~d}$ & $1,12 \mathrm{~d}$ \\
\hline ‘Rubi’ x (LVK x LCR - 038) & 0,0011 & $0,09 \mathrm{f}$ & $0,11 \mathrm{f}$ \\
\hline 'Rubi'x 'BRS Bravo' & $1,185 \mathrm{f}$ & $1,33 \mathrm{c}$ & $1,35 \mathrm{c}$ \\
\hline 'Pera'/CNPMF x 'Santa Cruz Rangpur' lime & $0,859 \mathrm{~h}$ & $1,01 d$ & $1,09 d$ \\
\hline 'Pera'/CNPMF x 'Sunki Tropical' & $1,345 \mathrm{e}$ & $1,35 \mathrm{c}$ & $1,41 \mathrm{c}$ \\
\hline 'Pera'/CNPMF x 'Indio' & $3,470 \mathrm{a}$ & $1,83 \mathrm{a}$ & $1,98 \mathrm{a}$ \\
\hline 'Pera'/CNPMF x 'Riverside' & $3,145 \mathrm{~b}$ & $1,92 \mathrm{a}$ & $1,85 \mathrm{a}$ \\
\hline 'Pera'/CNPMF x BRS Pompeu & $1,125 \mathrm{f}$ & $1,37 \mathrm{c}$ & $1,40 \mathrm{c}$ \\
\hline 'Pera'/CNPMF x (LVK x LCR - 038) & $0,795 \mathrm{~h}$ & $1,22 \mathrm{c}$ & $1,39 \mathrm{c}$ \\
\hline 'Pera'/CNPMF x 'BRS Bravo' & $3,430 \mathrm{a}$ & $1,69 b$ & $1,77 \mathrm{~b}$ \\
\hline 'Valência Tuxpan’ x 'Santa Cruz Rangpur’ lime & $0,785 \mathrm{~h}$ & $1,11 \mathrm{~d}$ & $1,23 \mathrm{~d}$ \\
\hline 'Valência Tuxpan’ x 'Sunki Tropical' & $1,516 \mathrm{e}$ & $1,45 \mathrm{c}$ & $1,57 \mathrm{c}$ \\
\hline 'Valência Tuxpan’ x 'Indio' & $1,886 \mathrm{~d}$ & $1,35 \mathrm{c}$ & $1,47 \mathrm{c}$ \\
\hline 'Valência Tuxpan’ x 'Riverside' & $1,446 \mathrm{e}$ & $1,26 \mathrm{c}$ & $1,36 \mathrm{c}$ \\
\hline 'Valência Tuxpan’ x BRS Pompeu & $0,549 \mathrm{i}$ & $1,10 \mathrm{~d}$ & $1,20 \mathrm{~d}$ \\
\hline 'Valência Tuxpan' x (LVK x LCR - 038) & $0,271 \mathrm{j}$ & $0,79 \mathrm{e}$ & $0,88 \mathrm{e}$ \\
\hline 'Valência Tuxpan’ x ‘BRS Bravo' & $1,386 \mathrm{e}$ & $1,31 \mathrm{c}$ & $1,38 \mathrm{c}$ \\
\hline 'Pineapple' x 'Santa Cruz Rangpur' lime & $0,582 \mathrm{i}$ & $1,05 d$ & $1,10 \mathrm{~d}$ \\
\hline 'Pineapple’ x 'Sunki Tropical' & $1,070 \mathrm{~g}$ & $1,31 \mathrm{c}$ & $1,35 \mathrm{c}$ \\
\hline 'Pineapple' x 'Indio' & $2,482 \mathrm{c}$ & $1,57 b$ & $1,61 b$ \\
\hline 'Pineapple' x 'Riverside' & $1,986 \mathrm{~d}$ & $1,51 \mathrm{c}$ & $1,55 \mathrm{c}$ \\
\hline 'Pineapple' x BRS Pompeu & $0,338 \mathrm{j}$ & $1,06 \mathrm{~d}$ & $1,11 d$ \\
\hline 'Pineapple' x (LVK x LCR - 038) & $0,568 \mathrm{i}$ & $1,08 \mathrm{~d}$ & $1,15 d$ \\
\hline 'Pineapple' x 'BRS Bravo' & $1,984 \mathrm{~d}$ & $1,58 \mathrm{~b}$ & $1,64 b$ \\
\hline F Test & $210.4070 * *$ & $12.0596 * *$ & $14.2532 * *$ \\
\hline Coefficient of variation (\%) & 14,23 & 22.20 & 19.43 \\
\hline
\end{tabular}

${ }^{1}$ TSKC: 'Sunk Comum' Tangerine [ C. sunk (Hayata) hort. Ex Tanaka]; CTSW: 'Citrumelo Swingle' (C. Paradise Macfad. x Poncirus Trifoliata); LVK: 'Volkameriano' Lemon Tree (C. volkameriana V. Ten. \& Pasq.); LCR: 'Rangpur' lime (C. Limonia Osbeck); TR: P. trifoliata (L.) Raf. ${ }^{2}$ Averages followed by the same letter in the column do not differ according to the Scott Knott test $(\mathrm{p}<0.05)$. 
Table 2. Cumulative yield (CY), scion volume (CV), and tree height (PH) of the combinations of seven rootstocks with four scions tested in the Manaus metropolitan region, AM, from 2017 to 2019.

\begin{tabular}{|c|c|c|c|}
\hline Combinations & CY (kg.plant $\left.{ }^{-1}\right)$ & $\mathrm{CV}\left(\mathrm{m}^{3}\right)$ & $\mathrm{PH}(\mathrm{m})$ \\
\hline 'Rubi’x 'Santa Cruz Rangpur' lime & $0,29 \mathrm{j}$ & $1,90 \mathrm{a}$ & $3,07 \mathrm{a}$ \\
\hline 'Rubi’ x 'Sunki Tropical' & 0,241 & $2,01 \mathrm{a}$ & $3,23 \mathrm{a}$ \\
\hline 'Rubi’ x 'Indio' & $1,82 \mathrm{f}$ & $2,10 \mathrm{a}$ & $3,42 \mathrm{a}$ \\
\hline 'Rubi'x 'Riverside' & $1,45 \mathrm{~g}$ & $1,91 \mathrm{a}$ & $3,07 \mathrm{a}$ \\
\hline 'Rubi' x BRS Pompeu & 0,161 & $1,47 \mathrm{~b}$ & $2,37 \mathrm{~b}$ \\
\hline ‘Rubi’ x (LVK x LCR - 038) & 0,001 & $1,29 \mathrm{~b}$ & $1,99 \mathrm{~b}$ \\
\hline 'Rubi’ x 'BRS Bravo' & $1,78 \mathrm{f}$ & $2,06 \mathrm{a}$ & $3,37 \mathrm{a}$ \\
\hline 'Pera'/CNPMF x 'Santa Cruz Rangpur' lime & $1,29 \mathrm{~h}$ & $1,77 \mathrm{a}$ & $2,78 \mathrm{~b}$ \\
\hline 'Pera'/CNPMF x 'Sunki Tropical' & $2,02 \mathrm{e}$ & $2,01 \mathrm{a}$ & $2,78 \mathrm{~b}$ \\
\hline 'Pera'/CNPMF x 'Indio' & $5,21 \mathrm{a}$ & $1,92 \mathrm{a}$ & $3,09 \mathrm{a}$ \\
\hline 'Pera'/CNPMF x 'Riverside' & $4,72 \mathrm{~b}$ & $1,89 \mathrm{a}$ & $3,09 \mathrm{a}$ \\
\hline 'Pera'/CNPMF x BRS Pompeu & $1,69 \mathrm{f}$ & $1,94 \mathrm{a}$ & $3,10 \mathrm{a}$ \\
\hline 'Pera'/CNPMF x (LVK x LCR - 038) & $1,19 \mathrm{~h}$ & $1,39 \mathrm{~b}$ & $2,06 \mathrm{~b}$ \\
\hline 'Pera'/CNPMF x 'BRS Bravo' & $5,14 \mathrm{a}$ & $2,03 \mathrm{a}$ & $3,30 \mathrm{a}$ \\
\hline 'Valência Tuxpan' x 'Santa Cruz Rangpur' lime & $1,18 \mathrm{~h}$ & $2,02 \mathrm{a}$ & $3,24 \mathrm{a}$ \\
\hline 'Valência Tuxpan'x 'Sunki Tropical' & $2,27 \mathrm{e}$ & $1,86 \mathrm{a}$ & $2,92 \mathrm{a}$ \\
\hline 'Valência Tuxpan' x 'Indio' & $2,83 \mathrm{~d}$ & $2,12 \mathrm{a}$ & $3,50 \mathrm{a}$ \\
\hline 'Valência Tuxpan'x 'Riverside' & $2,17 \mathrm{e}$ & $2,15 \mathrm{a}$ & $3,52 \mathrm{a}$ \\
\hline 'Valência Tuxpan'x BRS Pompeu & $0,82 \mathrm{i}$ & $1,90 \mathrm{a}$ & 3,06 a \\
\hline 'Valência Tuxpan’ x (LVK x LCR - 038) & $0,41 \mathrm{j}$ & $1,65 \mathrm{~b}$ & $2,61 \mathrm{~b}$ \\
\hline 'Valência Tuxpan’x ‘BRS Bravo’ & $2,08 \mathrm{e}$ & $2,07 \mathrm{a}$ & $3,46 \mathrm{a}$ \\
\hline 'Pineapple' x 'Santa Cruz Rangpur' lime & $0,87 \mathrm{i}$ & $2,13 \mathrm{a}$ & $3,51 \mathrm{a}$ \\
\hline 'Pineapple' x 'Sunki Tropical' & $1,61 \mathrm{~g}$ & $2,01 \mathrm{a}$ & $3,28 \mathrm{a}$ \\
\hline 'Pineapple' x 'Indio' & $3,72 \mathrm{c}$ & $2,05 \mathrm{a}$ & 3,33 a \\
\hline 'Pineapple' x 'Riverside' & $2,98 \mathrm{~d}$ & $2,03 \mathrm{a}$ & 3,39 a \\
\hline 'Pineapple' x BRS Pompeu & $0,51 \mathrm{j}$ & $1,84 \mathrm{a}$ & $3,00 \mathrm{a}$ \\
\hline 'Pineapple' x (LVK x LCR - 038) & $0,85 \mathrm{i}$ & $1,98 \mathrm{a}$ & $3,26 \mathrm{a}$ \\
\hline 'Pineapple' x'BRS Bravo' & $2,98 \mathrm{~d}$ & $1,97 \mathrm{a}$ & $3,21 \mathrm{a}$ \\
\hline F Test & $210.4070 * *$ & $2.9208 * *$ & $2,5525 * *$ \\
\hline Coefficient of variation (\%) & 14,23 & 17,64 & 21,83 \\
\hline
\end{tabular}

${ }^{1}$ TSKC: ‘Sunk Comum' Tangerine [ C. sunk (Hayata) hort. Ex Tanaka]; CTSW: 'Citrumelo Swingle' (C. Paradise Macfad. x Poncirus Trifoliata); LVK: 'Volkameriano' Lemon Tree (C. volkameriana V. Ten. \& Pasq.); LCR: 'Rangpur' lime (C. Limonia Osbeck); TR: P. trifoliata (L.) Raf. ${ }^{2}$ Averages followed by the same letter in the column do not differ according to the Scott Knott test $(\mathrm{p} \leq 0.05)$. 
Table 3. Total soluble solid (TSS) content, total titratable acidity (TTA), technological index (TI), juice content (JC), and ratio scion/rootstock combinations tested in the Manaus metropolitan region, AM, from 2017 to 2019.

\begin{tabular}{|c|c|c|c|c|c|}
\hline Combinations & TSS (\%) & TTA $(\%)$ & ratio & $\mathrm{JC}(\%)$ & TI \\
\hline 'Rubi’ x 'Santa Cruz Rangpur' lime & $9,16 \mathrm{a}$ & $1,05 \mathrm{c}$ & $9,81 \mathrm{e}$ & $25,54 \mathrm{~d}$ & $2,46 \mathrm{~d}$ \\
\hline 'Rubi’x 'Sunki Tropical’' & $5,95 \mathrm{c}$ & $0,30 \mathrm{f}$ & $13,16 \mathrm{c}$ & $25,54 \mathrm{~d}$ & $2,37 \mathrm{~d}$ \\
\hline 'Rubi’ x 'Indio' & $9,00 \mathrm{a}$ & $1,02 \mathrm{c}$ & $12,27 \mathrm{~d}$ & $36,17 \mathrm{c}$ & $3,22 \mathrm{c}$ \\
\hline 'Rubi'x 'Riverside' & $8,60 \mathrm{a}$ & $0,65 \mathrm{~d}$ & $14,50 \mathrm{c}$ & $47,43 \mathrm{~b}$ & $4,15 \mathrm{~b}$ \\
\hline ‘Rubi’ x BRS Pompeu & $2,96 \mathrm{e}$ & $0,16 \mathrm{f}$ & $6,02 \mathrm{f}$ & $16,48 \mathrm{e}$ & $1,46 \mathrm{e}$ \\
\hline ‘Rubi’ x (LVK x LCR - 038) & $2,77 \mathrm{e}$ & $0,12 \mathrm{f}$ & $6,45 \mathrm{f}$ & $15,38 \mathrm{e}$ & 1,49 e \\
\hline 'Rubi’ x ‘BRS Bravo' & $9,18 \mathrm{a}$ & 0,46 e & $20,84 \mathrm{a}$ & $45,22 \mathrm{~b}$ & $4,15 \mathrm{~b}$ \\
\hline 'Pera'/CNPMF x 'Santa Cruz Rangpur' lime & $7,45 \mathrm{~b}$ & $0,73 \mathrm{~d}$ & $9,39 \mathrm{e}$ & $40,49 \mathrm{~b}$ & $3,57 \mathrm{c}$ \\
\hline 'Pera'/CNPMF x 'Sunki Tropical' & $9,22 \mathrm{a}$ & $1,06 \mathrm{c}$ & $9,21 \mathrm{e}$ & $44,53 \mathrm{~b}$ & $4,10 \mathrm{~b}$ \\
\hline ‘Pera'/CNPMF x ‘Indio’' & $5,69 \mathrm{c}$ & $0,56 \mathrm{e}$ & $6,76 \mathrm{f}$ & $47,79 \mathrm{~b}$ & $4,03 \mathrm{~b}$ \\
\hline 'Pera'/CNPMF x 'Riverside' & $8,17 \mathrm{~b}$ & $0,76 \mathrm{~d}$ & $10,90 d$ & $44,40 \mathrm{~b}$ & $3,65 \mathrm{c}$ \\
\hline 'Pera'/CNPMF x BRS Pompeu & $5,64 \mathrm{c}$ & $0,58 \mathrm{e}$ & $6,55 \mathrm{f}$ & $33,39 \mathrm{c}$ & $2,80 \mathrm{~d}$ \\
\hline 'Pera'/CNPMF x (LVK x LCR - 038) & $5,94 \mathrm{c}$ & $0,67 \mathrm{~d}$ & $5,91 \mathrm{f}$ & $33,23 \mathrm{c}$ & $2,95 \mathrm{~d}$ \\
\hline 'Pera'/CNPMF x 'BRS Bravo' & $8,54 \mathrm{a}$ & $0,79 \mathrm{~d}$ & $11,31 \mathrm{~d}$ & $40,90 \mathrm{~b}$ & $3,49 \mathrm{c}$ \\
\hline 'Valência Tuxpan' x 'Santa Cruz Rangpur' lime & $7,70 \mathrm{~b}$ & $1,04 \mathrm{c}$ & $8,71 \mathrm{e}$ & $36,14 \mathrm{c}$ & $2,79 \mathrm{~d}$ \\
\hline 'Valência Tuxpan'x 'Sunki Tropical' & $6,91 \mathrm{~b}$ & $1,12 \mathrm{c}$ & $7,50 \mathrm{f}$ & $63,59 \mathrm{a}$ & $4,34 \mathrm{~b}$ \\
\hline 'Valência Tuxpan’ x 'Indio' & $7,99 \mathrm{~b}$ & $2,06 \mathrm{a}$ & $5,31 \mathrm{~g}$ & $46,15 \mathrm{~b}$ & $3,67 \mathrm{c}$ \\
\hline 'Valência Tuxpan’ x 'Riverside' & $5,77 \mathrm{c}$ & $0,56 \mathrm{e}$ & $9,33 \mathrm{e}$ & $23,95 \mathrm{~d}$ & $2,06 \mathrm{e}$ \\
\hline 'Valência Tuxpan'x BRS Pompeu & $4,80 \mathrm{~d}$ & $0,56 \mathrm{e}$ & $5,85 \mathrm{f}$ & $31,58 \mathrm{c}$ & $2,28 \mathrm{~d}$ \\
\hline 'Valência Tuxpan’ x (LVK x LCR - 038) & $4,65 \mathrm{~d}$ & $0,75 \mathrm{~d}$ & $3,98 \mathrm{~g}$ & $20,35 \mathrm{e}$ & $1,66 \mathrm{e}$ \\
\hline ‘Valência Tuxpan’x ‘BRS Bravo’ & $8,35 \mathrm{a}$ & $1,32 \mathrm{~b}$ & $7,97 \mathrm{e}$ & $44,50 \mathrm{~b}$ & $3,71 \mathrm{c}$ \\
\hline 'Pineapple' x 'Santa Cruz Rangpur' lime & $8,03 \mathrm{~b}$ & $0,82 \mathrm{~d}$ & $10,30 \mathrm{e}$ & $31,55 \mathrm{c}$ & $2,54 \mathrm{~d}$ \\
\hline 'Pineapple' x 'Sunki Tropical' & $8,06 \mathrm{~b}$ & $0,98 \mathrm{c}$ & $10,96 \mathrm{~d}$ & $36,78 \mathrm{c}$ & $2,94 \mathrm{~d}$ \\
\hline 'Pineapple’ x ‘'Indio’' & $7,73 \mathrm{~b}$ & $0,58 \mathrm{e}$ & $15,45 \mathrm{~b}$ & $34,70 \mathrm{c}$ & $2,71 \mathrm{~d}$ \\
\hline 'Pineapple' x 'Riverside' & $8,55 \mathrm{a}$ & $0,70 \mathrm{~d}$ & $13,47 \mathrm{c}$ & $29,80 \mathrm{c}$ & $2,54 \mathrm{~d}$ \\
\hline 'Pineapple' x BRS Pompeu & $8,76 \mathrm{a}$ & $0,68 \mathrm{~d}$ & $15,63 \mathrm{~b}$ & 59,19 a & $5,24 \mathrm{a}$ \\
\hline 'Pineapple' x (LVK x LCR - 038) & $8,57 \mathrm{a}$ & $0,63 \mathrm{e}$ & $13,96 \mathrm{c}$ & $28,69 \mathrm{c}$ & $2,47 \mathrm{~d}$ \\
\hline 'Pineapple' x 'BRS Bravo' & $9,14 \mathrm{a}$ & $0,95 \mathrm{c}$ & $11,69 \mathrm{~d}$ & $35,24 \mathrm{c}$ & $3,18 \mathrm{c}$ \\
\hline F Test & $36,90 * *$ & $25,49 * *$ & $44,27 * *$ & $17,14 * *$ & $15,37 * *$ \\
\hline Coefficient of variation (\%) & 11,16 & 25,42 & 15,22 & 20,21 & 20,27 \\
\hline
\end{tabular}

'TSKC: 'Sunk Comum' Tangerine [ C. sunk (Hayata) hort. Ex Tanaka]; CTSW: 'Citrumelo Swingle' (C. Paradise Macfad. x Poncirus Trifoliata); LVK: 'Volkameriano’ Lemon Tree (C. volkameriana V. Ten. \& Pasq.); LCR: Rangpur' Lime (C. Limonia Osbeck); TR: P. trifoliata (L.) Raf. ${ }^{2}$ Averages followed by the same letter in the column do not differ according to the Scott Knott test $(\mathrm{p} \leq 0.05)$. 


\section{Multivariate analysis}

We performed multivariate analyses for the varieties with better yield and quality parameters, to identify relatively homogeneous clusters of rootstocks combined with them. A PCA was applied to evaluate the variability and the relationships between the yield performance and fruit quality variables (Figure 1). For the rootstocks combined with 'Rubi,' the first two principal components explained $86.1 \%$ of the total variability found in the physical and chemical parameters; six main components explained total variability. For the scion variety 'Pera'/CNPMF, total data variability was explained by 4 principal components, and the first two explained $87.31 \%$ of the variability (Table 5). For 'Rubi,' the first component (PC1) was related to $\mathrm{CY}, \mathrm{CV}$, plant height, TSS content, TI, and JC, according to the contribution of the variables evaluated for each component (Table 4 and Figure 1). For the 'Pera'/CNPMF orange, the variables that contributed the most to the first component (PC1) were the CY, TSS content, ratio, and JC (Table 4 and Figure 1).
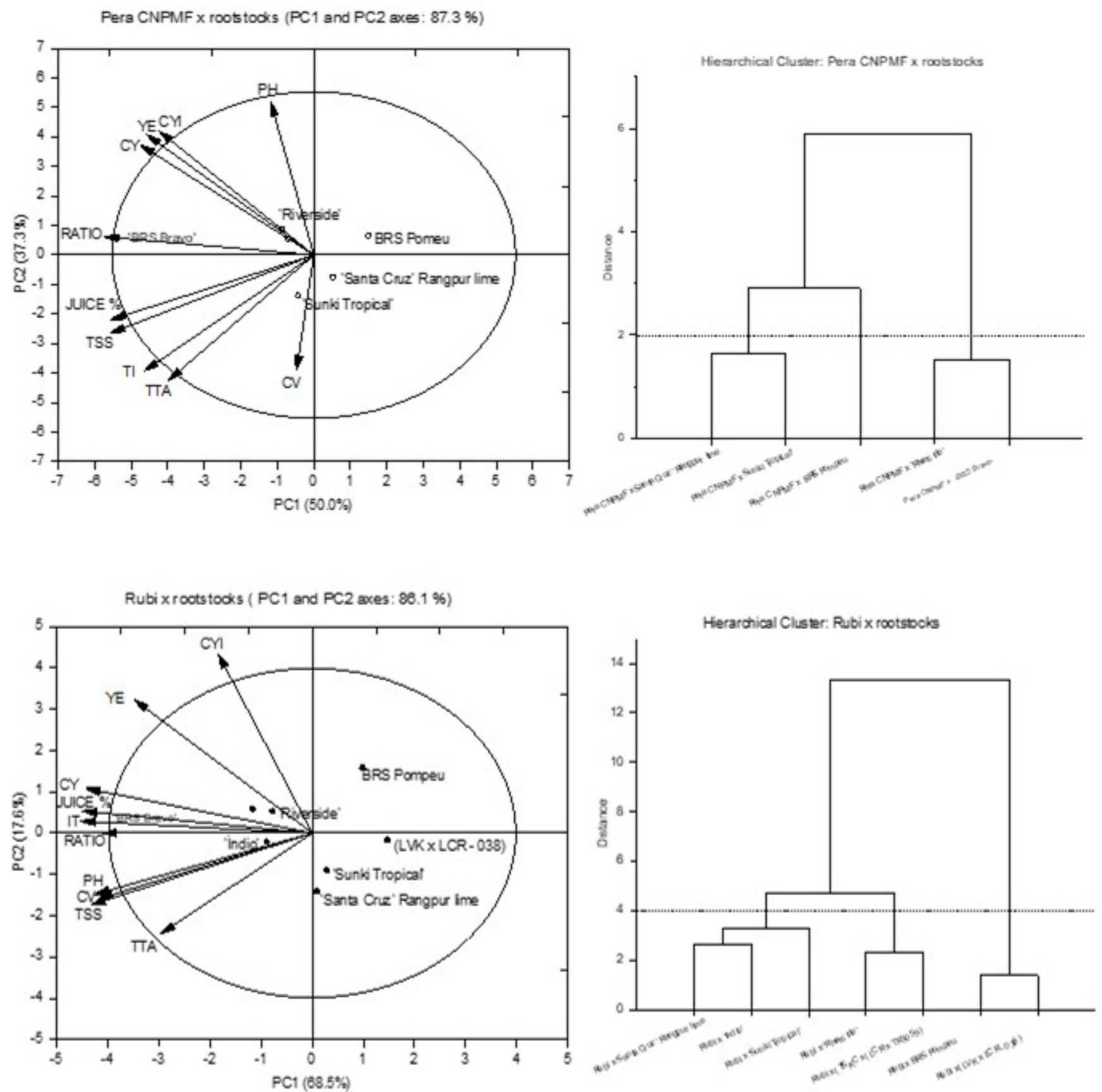

Figure 1. Principal Component Analysis (PCA), with correlation circle (95\% confidence ellipse), diagram of observations, and dendrogram of rootstock genotypes obtained by Ward's method, based on the Euclidean distance for orange varieties Rubi and Pera/CNPMF evaluated in 2017 to 2019. 
Table 4. Contribution of the variables evaluated for each component (factor loadings) in the Pera / CNPMF and 'Rubi' orange experiments in the Manaus metropolitan region, AM, from 2017 to 2019.

\begin{tabular}{cccc|ccc}
\hline \multirow{2}{*}{ Variables } & \multicolumn{3}{c}{ 'Pera'/CNPMF } & \multicolumn{3}{c}{ 'Rubi' } \\
\cline { 2 - 7 } & PC1 & PC2 & PC3 1 & PC2 & PC3 \\
\hline Yield Efficiency & $-0,3278$ & 0,34982 & $-0,0294$ & $-0,27907$ & 0,48433 & 0,25395 \\
Crop-Yield Index & $-0,30325$ & 0,3597 & $-0,23603$ & $-0,14704$ & 0,64989 & 0,2749 \\
Cumulative yield & $-0,33947$ & 0,31964 & 0,20378 & $-0,35508$ & 0,16238 & 0,0287 \\
Canopy volume & $-0,03398$ & $-0,33501$ & 0,75683 & $-0,33508$ & $-0,23866$ & $-0,03103$ \\
Tree height & $-0,08479$ & 0,44537 & 0,44894 & $-0,34104$ & $-0,22214$ & 0,00116 \\
Total soluble solids & $-0,39855$ & $-0,22655$ & 0,09881 & $-0,3467$ & $-0,26129$ & 0,15882 \\
Total titratable acidity & $-0,28418$ & $-0,36378$ & 0,01214 & $-0,23749$ & $-0,36572$ & 0,67491 \\
Ratio & $-0,41091$ & 0,05311 & 0,15224 & $-0,32911$ & $-1,10 \mathrm{E}-06$ & $-0,54045$ \\
Technological index & $-0,33219$ & $-0,33706$ & $-0,15585$ & $-0,36209$ & 0,04378 & $-0,21875$ \\
Juice Content & $-0,39775$ & $-0,18813$ & $-0,2649$ & $-0,36028$ & 0,08034 & $-0,19362$ \\
\hline
\end{tabular}

Table 5. Eigenvalues of principal component analysis for orange varieties evaluated without experience of seven rootstocks in combination with four copies in the Manaus metropolitan region, AM, from 2017 to 2019.

\begin{tabular}{ccccc}
\hline Canopy & $\begin{array}{c}\mathbf{n}^{\mathbf{0}} \text { Principal } \\
\text { Component }\end{array}$ & Eigenvalue & $\begin{array}{c}\text { Percentage of } \\
\text { Variability }(\mathbf{\%})\end{array}$ & $\begin{array}{c}\text { Cumulative } \\
\mathbf{( \% )}\end{array}$ \\
\hline \multirow{3}{*}{ 'Rubi' } & $\mathbf{1}$ & $\mathbf{6 , 8 5 4 8 8}$ & $\mathbf{6 8 , 5 4 8 7 8}$ & $\mathbf{6 8 , 5 4 8 7 8}$ \\
& $\mathbf{2}$ & $\mathbf{1 , 7 5 6 0 5}$ & $\mathbf{1 7 , 5 6 0 5 3}$ & $\mathbf{8 6 , 1 0 9 3 1}$ \\
& 3 & 0,79084 & 7,90845 & 94,01775 \\
& 4 & 0,38808 & 3,8808 & 97,89856 \\
& 5 & 0,16166 & 1,61659 & 99,51515 \\
& 6 & 0,04849 & 0,48485 & 100 \\
\hline \multirow{2}{*}{ 'Pera/CNPMF' } & $\mathbf{1}$ & $\mathbf{5 , 0 0 3 4 4}$ & $\mathbf{5 0 , 0 3 4 4 2}$ & $\mathbf{5 0 , 0 3 4 4 2}$ \\
& $\mathbf{2}$ & $\mathbf{3 , 7 2 8 4 2}$ & $\mathbf{3 7 , 2 8 4 2 1}$ & $\mathbf{8 7 , 3 1 8 6 3}$ \\
& 3 & 1,00034 & 10,0034 & 97,32203 \\
& 4 & 0,2678 & 2,67797 & 100 \\
\hline
\end{tabular}

Hierarchical Cluster Analysis showed four distinct clusters of rootstocks in combination with the 'Rubi' scion. The first group was formed by 'Rangpur' lime and 'Indio' citrandarin. The 'Riverside' and the 'BRS Bravo' citrandarins formed the second homogeneous cluster, whose Euclidean distance was similar to that of the rootstocks of the first cluster (Figure 1). For 'Pera'/ CNPMF, three rootstock clusters were found. The 'Rangpur' lime and 'Sunki Tropical' rootstocks formed the first cluster, and the 'Riverside' and TSKS x 'BRS Bravo' citrandarins also showed a similar Euclidean distance, compared to the first group rootstocks. Importantly, in the PCA for the 'Pera'/CNPMF (Figure 1). PCA showed that the 'BRS Bravo' , 'Riverside,' 'Indio,' and 'Sunki Tropical' rootstocks had similar levels of performance for CY, YE, and CYI when combined with 'Pera'/CNPMF, 'Pineapple,' 'Rubi,' and 'Valencia Tuxpan' scions (Figure $1)$.

\section{Production, cumulative yield, yield efficiency, and crop-yield index}

The 2017 to 2019 CY of 'Indio,' 'BRS Bravo', and 'Riverside' rootstocks combinations were among the best even for scions with lower yield performances, such as 'Valencia Tuxpan' and 'Pineapple' (Table 1). Rodrigues (2019), in the State of Acre (BR), reported that the LVK x LCR - 038, 'Indio' citrandarin, and 'Rangpur' lime rootstocks increased the CY of 'Pera'/CNPMF reaching values between 180 and $190 \mathrm{~kg} /$ tree. The 'Indio', 'BRS Bravo', and 'Riverside' rootstocks had better performance than the 'Rangpur' lime rootstock combined with the 'Pera'/CNPMF scion. Carvalho et al. (2019) obtained a higher yield with the 'Valencia Tuxpan' scion grafted onto 'Rangpur' lime. These authors also reported that this combination achieved the highest $\mathrm{CY}$ for the six years of the study. 
Better yield performances per tree of 'Pera' orange grafted onto 'Indio' and 'Riverside' rootstocks were also reported by Sampaio et al. (2016) and by França et al. (2016) in the state of Bahia (BR). Mitra (2018) reported that different citrus rootstocks varied in their drought tolerance. Somatic hybrid tetraploid rootstocks have been used to combine plants resistant to biotic and abiotic stresses, including drought, salinity, and alkalinity, and abiotic stress by pathogens. Environmental and agronomic conditions, such as light, temperature, plant nutrition, and rootstock selection, among others, also influence drought tolerance (LADO et al., 2018). Better canopy-rootstock combinations can be selected for each production ecosystem, and drought tolerance is a fundamental trait in rainfed agriculture. This may explain the critical influence of rootstocks in drought periods in the Amazon. Therefore, results indicate that the 'Riverside' and 'Indio' citrandarins induced greater production and productive efficiency in the 'Pera' orange. It is noteworthy that these rootstocks are considered as alternatives to the 'Carnation' lemon for the combination with the sweet orange 'Pera' under drought conditions. This is crucial for the Amazon drought conditions.

During water stress, the plant root system must decrease its water potential below the water potential of the soil to absorb water, through having high hydraulic conductance, deep and dense roots, and protoplasmic tolerance. This might explain the greater cumulative productivity of 'Indio,' 'BRS Bravo', and 'Riverside' combined with the 'Pera'/CNPMF, which is already well adapted to the tropical Amazon region dry season. The resistance of those rootstocks to drought and other stresses was confirmed by the increased production year-round. Miranda et al. (2021) showed that the root hydraulic redistribution, the osmotic adjustment, and the stomatal control of gas exchange in the leaf are important physiological mechanisms associated with the drought tolerance induced by 'Carnation' and 'Swingle' citrumelo rootstocks. These data show the high physiological adaptability of the rootstock 'Indio,' 'Riverside,' and 'BRS Bravo' to the high and low rainfall conditions of the Amazon and the consequent induction of higher production.

\section{Fruit quality}

Fruit quality is inherent to scions and is affected by rootstocks (SIQUEIRA and SALOMÃO, 2017). According to BASTOS et al. (2014), the 'Ruby' scion stands out in the fresh fruit market due to its seedless fruit, mid-season fruit ripening, medium juice yield, and medium acidity. Our results show that the 'Ruby' scion had the highest ratio, suggesting that in addition to having a better quality, its fruits matured earlier than for the other combinations. Nawaz et al. (2020) found that the TSS content increases throughout fruit development, which results in higher maturation and, consequently, a higher ratio, a parameter that indicates the point of commercial maturity. Hence, the lower the TTA in relation to TSS content, the higher the ratio, and faster the maturation. For Siqueira and Salomão (2017), low acidity allows fruits to be harvested earlier (because they ripen earlier) if they reach a $40 \% \mathrm{JC}$ and become suitable for consumption, even if their peel is green. The 'BRS Bravo', 'Indio,' and 'Riverside' rootstocks combined with 'Rubi' induced different fruit quality and always performed better than the others in TSS content, TTA, ratio, JC, and TI. The 'Pera'/CNPMF x 'Sunki Tropical' combination resulted in the highest fruit TSS content (Table 3). This result was corroborated by the findings of Cruz (2019) in Bahia.

'Pera' orange fruits have 1.5 to $1.4 \%$ TTA and 10 to $11^{\circ}$ Brix TTA (Lemos et al., 2012). These values are similar to those of our 'Pera'/CNPMF $\mathrm{x}$ 'Sunki Tropical' combinations. These same authors also found high JC values of approximately $60 \%$. For this scion, the rootstock influence on quality characteristics was also evident, as confirmed by Amorim et al. (2018), in which several citrus scions and six rootstock combinations were tested in northern Bahia, reaching similar results to our 'Pera'/CNPMF x 'Sunki Tropical' combination (Table 3). Rodrigues (2018) found that 'Valencia' orange fruits had higher TSS in combination with the LVK x LCR - 038, 'Rangpur' lime, and 'Indio' citrandarin rootstocks in the Acre state. Additionally, this scion in combination with 'Indio' had similar values to those of the combination 'Valencia Tuxpan' x 'BRS Bravo' in the local Amazonas conditions.

It is also worth emphasizing the importance of the Amazonian dry season and the influence of rootstock 'BRS Bravo' on fruit quality. Robles et al. (2017) demonstrated that alemow (Citrus macrophylla Wester.) and sour orange (Citrus aurantium L.) significantly increase TSS content and ATT in 'Verna' lemon 51 under regulated deficient irrigation conditions. This shows that some rootstocks can increase fruit TSS accumulation under water stress.

\section{Multivariate analysis}

PCA separated the physiological and biochemical variables that contributed to greater drought tolerance of scion/rootstock combinations, showing that 'Rangpur' lime and 'Sunki Tropical' mandarin behaved similarly under water stress. These rootstocks had higher antioxidant capacity because of enhanced root peroxidase activity; this may result from an increase in gene expression specific to rootstocks (SANTOS et al., 2019). These results highlight the influence of rainfall variation on orange fruit production and quality throughout the year, as the period with the lowest rainfall occurs from June to August in the Manaus metropolitan region. During these 
months, the grafted trees were under low soil humidity conditions, which probably caused an increase in fruit TSS concentration, increasing the maturation index.

Santos et al. (2019) reported similar results for 'Rangpur' lime and 'Sunki Tropical' which was confirmed in the 'Pera' and 'Rubi' scion PCA (Figure 1). However, the fruit quality variables, TSS content, TTA, and ratio, differed for the 'Rangpur' lime rootstock in comparison to 'Sunki Tropical, 'BRS Bravo', 'Riverside,' and 'Indio' (Figure 1). These results indicate that the relatively homogeneous rootstock clusters may behave differently, depending on the sweet orange variety used (CARVALHO et al., 2019).

\section{Conclusion}

The 'Indio, 'BRS Bravo', and 'Riverside' citrandarin rootstocks were more adapted to Amazonian conditions and provided the highest fruit production per tree when combined with 'Pera'/CNPMF orange scions. The rootstock 'BRS Bravo' led to the best fruit quality in the 'Rubi' and 'Pineapple' scions. Although the 'Rubi' and 'Pineapple' scions had the best fruit quality, the combinations 'Pera'/CNPMF scion with 'BRS Bravo', 'Índio,' and 'Riverside' were the most productive and also had good fruit quality. Therefore, these combinations will potentially be the most suitable for the Amazon region.

\section{Acknowledgements}

We would like to thank Fundação de Amparo à Pesquisa do Estado do Amazonas (FAPEAM), Universidade Federal do Amazonas (UFAM), Empresa Brasileira de Pesquisa Agropequaria (Embrapa), and Coordenação de Aperfeiçoamento de Pessoal de Nível Superior (CAPES).

\section{References}

AMORIM, M. da S.; GIRANDI, E.A.; FRNAÇA, N.O.; GESTEIRA, A.S.; FILHO, W.S.S.; PASSOS, O.S. Initial Performance of Alternative Citrus Scion and Rootstock Combinations on the Northern Coast of the State of Bahia, Brazil. Revista Brasileira de Fruticultura, Jaboticabal, v.40, n.4, p.1-10. 2018.

BASTOS, D. C.; FERREIRA, E. A.; PASSOS, O. S. Cultivares copa e porta-enxertos para a citricultura brasileira. Informe Agropecuário, Belo Horizonte, v.35, n.281, p.36-45, 2014.
BLUMER, S. Citrandarins e outros híbridos de trifoliata como porta-enxertos nanicantes para a laranjeira 'Valência' (Citrus sinensis L. Osbeck). 2005. Tese (Doutor em Agronomia) - Escola Superior de Agricultura "Luiz de Queiroz", Universidade de São Paulo, Piracicaba, 2005.

CARVALHO, L. M. D.; CARVALHO, H.W.L.; BARROS, I.; MARTINS, C.R.; FILHO, W.S.S.; GIRARDIE.A.; PASSOS, O.S. New scion-rootstock combinations for diversification of sweet orange orchards in tropical hardsetting soils. Scientia Horticulturae, New York, v.243, p.169-176, 2019.

CARVALHO, L. M.; CARVALHO, H.W.L; FILHO, W.S.S.; MARTINS, C.R.; PASSOS, O.S. Porta-enxertos promissores, alternativos ao limoeiro 'Cravo,' nos Tabuleiros Costeiros de Sergipe. Pesquisa Agropecuária Brasileira, Brasília, DF, v.51, n.2, p.132-141, 2016.

CHITARRA, M. I. F.; CHITARRA, A. B. Pós-colheita de frutas e hortaliças: fisiologia e manuseio. Lavras: UFLA, 1990.

CRUZ, E.S. Caracterização de porta-enxertos alternativos de citros sob diferentes regimes hídricos: relações hídricas, sistema radicular e influência sobre a produção e qualidade de frutos de laranja 'Pera' (Citrus sinensis L. Osb.). 2019. Tese (Doutor em Produção Vegetal) - Universidade Estadual de Santa Cruz , Ilhéus, 2019.

FRANÇA, N.O.; AMORIM, M.S.; GIRARD, E.A.; PASSOS, O.S.; FILHO,W.S.S. Desempenho da laranjeira 'Valência Tuxpan' sobre 14 porta-enxertos no litoral norte da Bahia. Revista Brasileira de Fruticultura, Jaboticabal, v.38, n.4, e.684, 2016.

IBGE - Instituto Brasileira de Goegrafia e Estatística. Levantamento sistematico da produção agrícola. Rio de Janeiro, 2019. Disponível em: https://sidra.ibge.gov. br/pesquisa/lspa/tabelas. Acesso em: 26 jul. 2019.

LADO, J.; GAMBETTA, G.; ZACARIAS, L. Key determinants of citrus fruit quality: Metabolites and main changes during maturation. Scientia Horticulturae, New York, v.233, p.238-248, 2018. 
MIRANDA, M.T.; SILVA, S.F.; SILVEIRA, N.M.; PEREIRA, L.; MACHADO, E.C; RIBEIRO, R.V. Root osmotic adjustment and stomatal control of leaf gas exchange are dependent on citrus rootstocks under water deficit. Journal of Plant Growth Regulation, New York, v.40, p.11-19, 2021.

MITRA, S. K. Climate change: impact, and mitigation strategies for tropical and subtropical fruits. Acta Horticulturae, The Hague, v.1216, p.1-12, 2016.

NAWAZ, R.; ABASSI, N.A.; HAFIZ, I.A.; KHALID, A. Impact of climate variables on fruit internal quality of Kinnow mandarin (Citrus nobilis Lour x Citrus deliciosa Tenora) in ripening phase grown under varying environmental conditions. Scientia Horticulturae, New York, v.265, p.109235, 2020.

NEGREIROS, J. R. da S.; NETO, R.C.A.; MIQUELONI, LESSA, L.S. Estimativa de repetibilidade para caracteres de qualidade de frutos de laranjeira-doce. Pesquisa Agropecuaria Brasileira, Brasília, DF, v.49, n.1, p.40-48, 2014.

PETRY, H. B.; REIS, B.; SILVA, R.R.; GONZATO, M.P.; SCHWARZ, S.F. Porta-enxertos influenciam o desempenho produtivo de laranjeiras-de-umbigo submetidas a poda drástica. Pesquisa Agropecuaria Tropical, Goiânia, v.45, n.4, p.449-455, 2015.

RIBEIRO, L.O.; GIRARD, E.A.; CARVAHO, H.W.L.; BASTS, D.C.; FILHO, W.S.S.; PASSOS, O.S. Evaluation of mandarin hybrids grafted on 'Rangpur' lime in the Coastal Tablelands, Brazil. Revista Brasileira de Fruticultura, Jaboticabal, v.42, n.6, 2020.

ROBLES, J. M.; BOTÍA, P.; PÉREZ-PÉREZ, J. G. Sour orange rootstock increases water productivity in deficit irrigated 'Verna' lemon trees compared with Citrus macrophylla. Agricultural Water Management, New York, v.186, p.98-107, 2017.
RODRIGUES, J.D.B.; MOREIRA, A.S.; STUCHI, E.S.; BASSANEZI, R.B.; LARANJEIRA, F.F.; GIRARDI, E.A. Huanglongbing incidence, canopy volume, and sprouting dynamics of 'Valencia' sweet orange grafted onto 16 rootstocks. Tropical Plant Pathology, Brasília, DF, v.45, n.6, p.611-619, 2020.

RODRIGUES, M. J. S. Desempenho de laranjeiras 'Pera'e 'Valência' sobre diferentes porta-enxertos, em Rio Branco, Acre. 2018. Tese (Doutor em Agronomia) - Universidade Federal do Acre, Rio Branco, 2018.

RODRIGUES, M. J. S.; NETO, S.E.A.; NETO, R.C.A; FILHO, W.S.S.; GIRARD, E.A.; LESSA, L.S.; ALMEIDA, U.O.; ARAÚJO, J.M. Agronomic performance of the 'Pera' orange grafted onto nine rootstocks under the conditions of Rio Branco, Acre, Brazil. Revista Brasileira de Ciências Agrárias, v.14, n.4, p.e6642, 2019.

SAMPAIO, A. H. R.; FILHO, M.A.C.; SOUZA, L.D.; BRITO, R.B.F.; SILVA, R.O. Produção e qualidade de frutos da laranjeira - 'pera' em combinação com diferentes porta-enxertos em condições de sequeiro. Revista Brasileira de Fruticultura, Jaboticabal. v.38, n.3, p.1-9, 2016.

SAMPAIO, A.H.R.; SILVA, R.O.; BRITO, R.B.F.; FILHO, W.S.S.; GESTEIRA, A.S.; SOUZA, L.D.; FILHO, M.A.C. Sweet orange acclimatisation to water stress: a rootstock dependency. Scientia Horticulturae, New York, v.276, p.109727, 2021.

SANTOS, I. C. DOS.; DEALMEIDA, A.A.F.; PIROVANI, C.P.; COSTA, M.G.C.; CONCEIÇÃO, A.S.; FILHO, W.S.S.; FILHO, M.A.C.; GESTEIRA, A.S. Physiological, biochemical and molecular responses to drought conditions in field-grown grafted and ungrafted citrus plants. Environmental and Experimental Botany, Amsterdam, v.162, p.406-420, 2019.

SIQUEIRA, D. L.; SALOMÃO, LCC. Citros do plantio à colheita. Viçosa: UFV, 2017. 